\title{
Le carcinome in situ du testicule - un précurseur du cancer à cellule germinale. Revue de la pathogenèse et aspects cliniques.
}

\author{
E.RAPJERT-DE MEYTS, A.GIWERCMAN, N.E.SKAKKEBAEK \\ Department of Growth and Reproduction, Copenhagen University Hospital, Copenhagen, Denmark.
}

\section{RÉSUMÉ}

Les tumeurs à cellule germinale du testicule diffèrent des autres tumeurs solides en ce qui concerne leur épidémiologie et certains aspects biologiques. La pathogenèse de ces tumeurs malignes est encore inconnue à l'exception du fait que tous les types de tumeurs à cellule germinale des adultes jeunes proviennent d'un précurseur commun : la cellule du carcinome in situ (CIS). Une très forte augmentation récente de l'incidence du cancer du testicule suggère que des facteurs environnementaux, plus particulièrement ceux qui agissent au cours du développement, pourraient jouer un rôle. Cette revue résume les caractéristiques biologiques les plus importantes du CIS du testicule et discute sa prise en charge clinique.

Mots clés : Néoplasmes testiculaires, carcinome in situ, cellule germinale, développement gonadique, reproduction masculine.

\section{INTRODUCTION}

Le cancer testiculaire à cellule germinale constitue l'affection maligne la plus commune chez les hommes âgés de 25 à 40 ans. Pour des raisons inconnues, au cours de la seconde moitié du vingtième siècle, on a observé une augmentation, qui se poursuit, de 3 à 4 fois de l'incidence de cette affection maligne. Cette augmentation a été constatée dans de nombreux pays ; il apparaît cependant des différences géographiques frappantes (figure 1) [1]. L'incidence spécifique de l'âge et d'autres données cliniques démontrent au-delà de tout doute qu'en dépit des variations géographiques, cette maladie est la même dans tous les pays et qu'elle possède fort probablement la même pathogenèse. La grande majorité des tumeurs testiculaires à cellule germinale surviennent chez les adolescents et les adultes jeunes. Ces tumeurs présentent au plan histologique une grande diversité de formes qui sont sommairement regroupées en tumeurs séminomateuses et non séminomateuses. Ces tumeurs sont toutes précédées par un carcinome in situ (CIS) non invasif qui semble luimême provenir, au cours de la vie foetale ou périnatale, d'une cellule germinale primitive $[2,3]$. Il existe, en outre, de rares tumeurs à cellule germinale dans d'autres groupes d'âge : tératomes et tumeurs du sac vitellin chez l'en-

Correspondance : Ewa Rapjert-De Meyts,

Department of Growth and Reproduction, Rigshospitalet, Section GR-5064, 9 Blegdamsvej

DK-2100 Copenhagen, Denmark. E mail : erm@rh.dk 
fant et séminomes spermatocytaires chez l'homme plus âgé. Ces tumeurs rares ne sont pas précédées par un CIS, leur incidence n'a pas augmenté, aussi leur pathogenèse est apparemment différente. Seule est envisagée dans cette revue la pathogenèse des tumeurs à cellule germinale de l'adulte jeune qui proviennent de cellules du CIS.

\section{HISTOPATHOLOGIE ET ASPECTS MOLÉCULAIRES DU CIS}

Dans les cas typiques, dans les testicules post pubertaires, les cellules du CIS sont trouvées à l'intérieur des tubules séminifêres, le long de la membrane basale du tubule, avec les cellules de Sertoli comme seul autre type cellulaire présent (Figure 2). On observe très rarement une spermatogenèse dans les tubules contenant un CIS. Les tubules avec CIS sont habituellement dispersés dans le testicule et ils peuvent représenter d'un faible pourcentage à pratiquement $100 \%$ des tubules. Un signe de croissance invasive précoce peut être observé de temps en temps sous la forme d'une dissémination du CIS dans le tissu interstitiel ou dans les cellules du rete testis. Le CIS est communément accompagné d'une infiltration lymphocytaire interstitielle.

Les cellules du CIS possèdent habituellement un volumineux noyau irrégulier avec des amas grossiers de chromatine et un cytoplasme abondant. La morphologie des cellules du CIS n'est pas identifiable dans les cas où le CIS apparaît comme un phénomène isolé précédant le développement de la tumeur ou lorsque le CIS est trouvé dans le tissu, d'apparence macroscopiquement normale, adjacent aux tumeurs déclarées, ce qui semble être le cas dans presque $100 \%$ de ces tumeurs $[4,5]$.

Dans de rares cas, comme chez les individus ayant une ambiguïté sexuelle et chez les garçons ayant une orchidopexie pour cryptorchidie, le CIS a été retrouvé dans des testicules d'enfants [6-8]. Dans les testicules d'enfants, la morphologie des cellules du CIS ressemble à celle des cellules du CIS de l'adulte, bien que les cellules malignes soient dispersées au sein de cellules de Sertoli immatures. Le nombre de cellules du CIS dans le testicule pré-pubère est habituellement plutôt faible, et comme ces cellules ressemblent à des gonocytes infantiles sous de nombreux aspects, le CIS pré-pubère peut être de diagnostic difficile.

Les cellules du CIS partagent de nombreuses caractéristiques avec les séminomes et - à un moindre degré - avec le carcinome embryonnaire. Il existe aussi une étroite relation immunohistochimique entre le CIS et les tumeurs déclarées qui expriment fréquemment des marqueurs similaires. En pratique clinique, le marqueur le plus utilisé est la phosphatase alcaline placentaire like (PLAP) (figure 3) $[9,10]$, les autres marqueurs ayant une signification histologique sont représentés par le proto-oncogène c-kit (Kit) $[11,12]$. Le produit de la protéine c-kit, Kit, qui est un récepteur pour le stem cell factor (SCF), est aussi fortement exprimé par les cellules germinales foetales jeunes (jusqu'à 12 semaines de gestation humaine) et par les gonocytes infantiles, mais pas par les cellules germinales de la période tardive de la gestation ni par les spermatogonies adultes normales, à l'exception des cas d'ambiguïtés sexuelles [13,14].

Ont été aussi rapportés comme marqueurs du CIS [15-17] plusieurs anticorps dirigés contre des glycolipides et glycoprotéines dont la fonction est encore inconnue, comme Gb-3, TRA-160 et M2A. IL est cependant important de se rappeler que les cellules du CIS sont fréquemment hétérogènes et que les marqueurs immunohistochimiques peuvent n'être exprimés que dans une sous-population de cellules du CIS seulement, en particulier dans les cas de CIS adjacents à des tumeurs mixtes [18].

Les mesures d'ADN sur CIS montrent le caractère malin de ces cellules qui ont un contenu en $\mathrm{ADN}$ aneuploïde, à la limite de la tétraploïdie [19]. Jusqu'à présent, très peu de modifications chromosomiques spécifiques ont été retrouvées dans les tumeurs à cellule germinale, à l'exception notable d'un iso chromosome sur le bras court du chromosome $12 \mathrm{i}(12 \mathrm{p})$ [20]. Cette aberration s'est avérée être la modification chromosomique la plus consistante et la plus spécifique retrouvée dans tous les types de tumeurs à cellule germinale [21]. Des tumeurs qui sont $\mathrm{i}(12 \mathrm{p})$ négatives ont été sporadiquement rapportées, mais même dans ces cas des réarrangements ou une sur-représentation du chromosome 12 ont été détectées [22]. 
Incidence of Testicular Cancer (per 100.000)

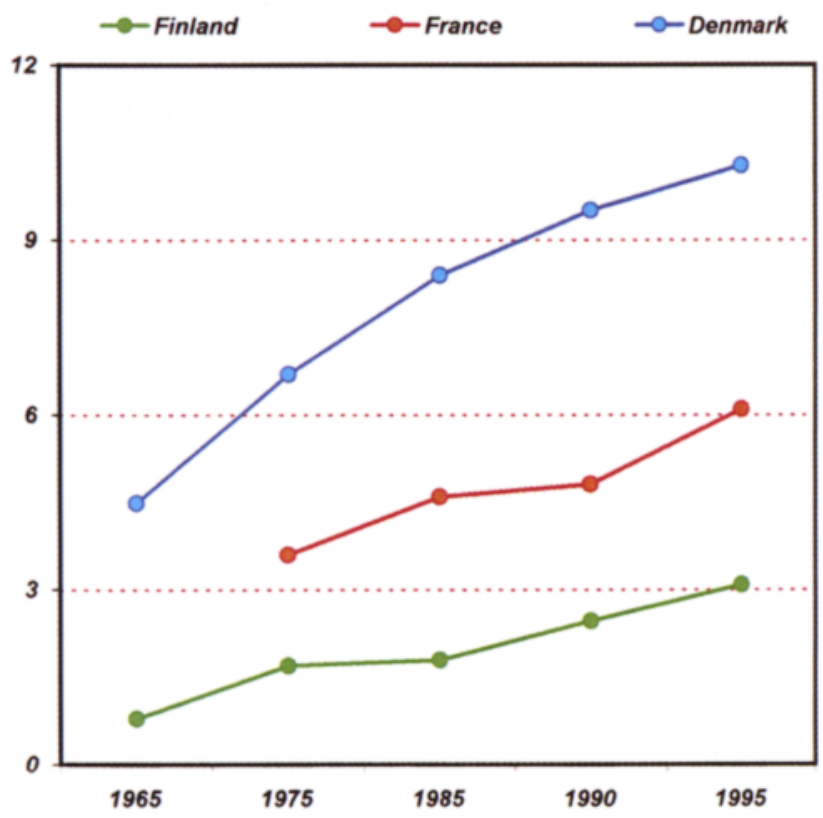

Figure 1. Evolution des taux d'incidence du cancer du testicule dans certains pays (compilation des références [1] et [89]].
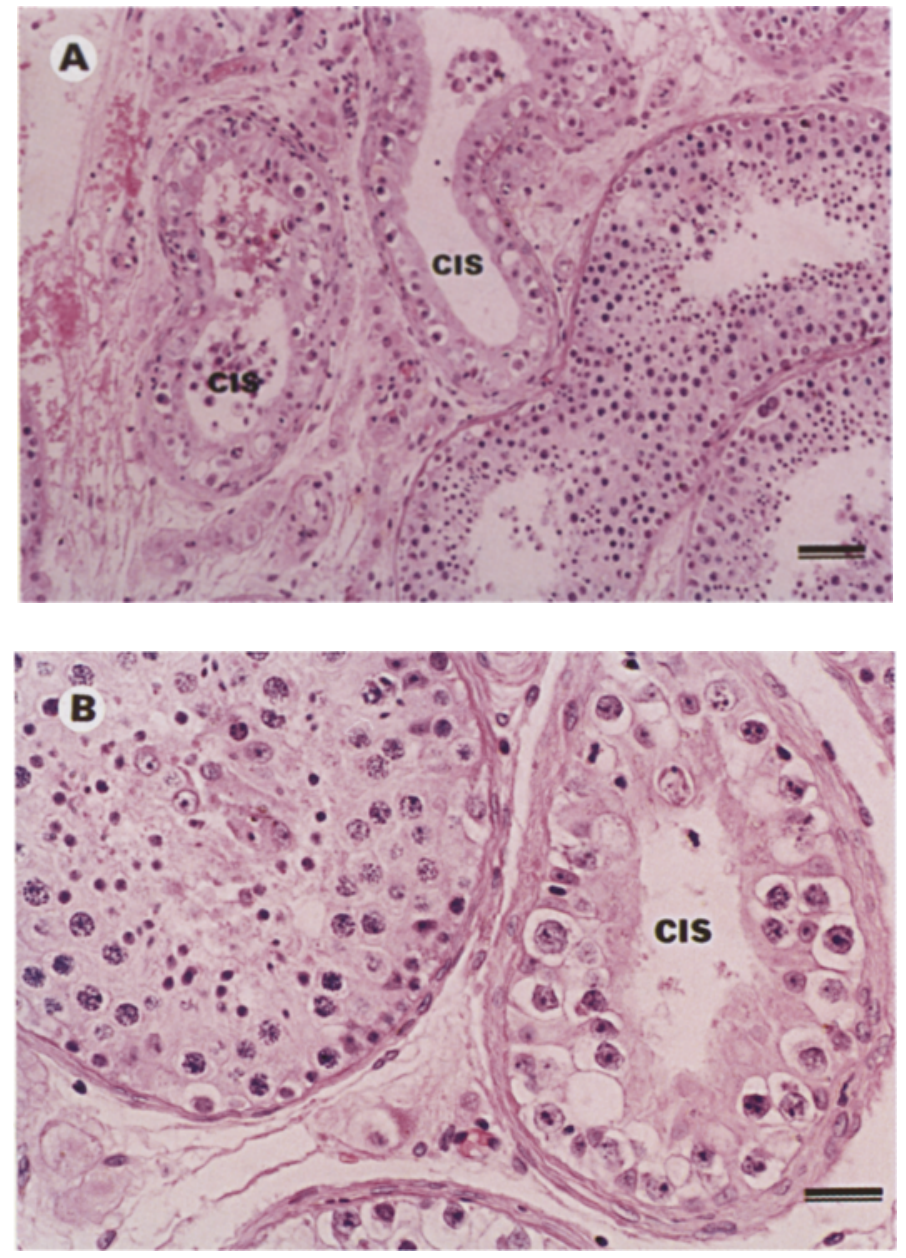

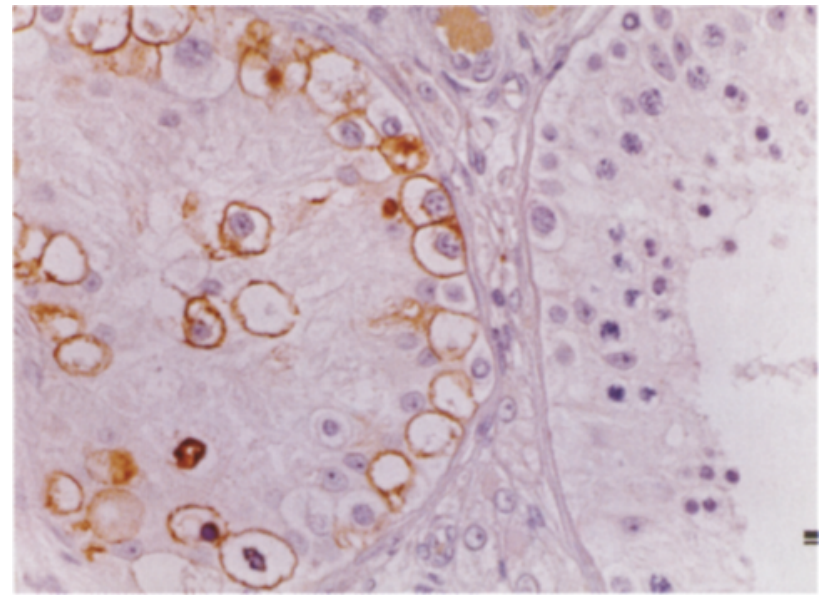

Figure 3. Marquage à l'immuno-peroxidase de cellules de carcinome in situ au moyen d'un anticorps dirigé contre la phosphatase alcaline placentaire like (PLAP), un marqueur du CIS cliniquement utile. Notez que les cellules germinales non malignes dans le tubule adjacent, qui présente une spermatogenèse en cours, ne sont pas colorées. Barre $=25 \mu \mathrm{m}$.

Figure 2. Biopsie testiculaire montrant un carcinome in situ (CIS).

A. Faible grossissement (barre $=50 \mu \mathrm{m}$ ). Remarquez les tubules renfermant le CIS par rapport aux tubules adjacents ayant une spermatogenèse conservée.

B. Plus fort grossissement (barre $=25 \mu \mathrm{m}$ ). Les cellules du CIS présentent les caractéristiques cytologiques de la malignité, à savoir de volumineux noyaux irréguliers avec une chromatine grossière. 
Contrairement au grand nombre d'études cytogénétiques sur les tumeurs invasives du testicule, il existe très peu de travaux sur la nature génétique des cellules du CIS. La raison principale en est la rareté du matériel biologique et l'absence de capacité des cellules du CIS de survivre en milieu de culture. Des études cytogénétiques conventionnelles de l'interphase indiquent que le nombre de copies du chromosome 15 est moindre dans les cellules du CIS adjacentes à une tumeur non séminomateuse que dans celles adjacentes à un séminome [23]. Liiso chromosome $12 p$ n'a été que sporadiquement retrouvé dans les cellules du CIS et sa signification pathogénique reste discutée [24].

Les récents progrès de la biologie moléculaire ont ouvert de nouvelles possibilités d'étude de l'implication des proto-oncogènes et des supresseurs tumoraux potentiels dans les tumeurs à cellule germinale [25]. Plusieurs groupes sont centrés sur le chromosome 12 car la présence de $\mathrm{i}(\mathrm{p} 12)$ conduit à une relative sur-expression des gènes situés sur le bras court par rapport à la sous-représentation du bras long [26]. Parmi ces gènes, la cycline D2 (localisée sur 12p) est sur-exprimée dans le CIS et apparaît être un bon candidat pour un oncogène putatif [27]. Cependant, la forte expression de la cycline D2 dans les cellules du CIS pourrait simplement refléter leur génotype gonocyte-like [28]. Les cyclines de type D sont impliquées dans la régulation de la protéine du rétinoblastome ( $\mathrm{RB}$ ) dans le cycle cellulaire. Une expression diminuée de l'ARNm du gène $\mathrm{RB}$ a été rapportée dans les tumeurs à cellule germinale avec une absence virtuelle de protéine $\mathrm{RB}$ dans le CIS et le séminome, et avec une augmentation importante de son expression dans les tumeurs plus différenciées [29]. Le rôle d'une forte expression de la cycline D2 dans ce contexte reste à élucider. L'expression des autres cyclines dans les cellules du CIS n'est pas différente de celle retrouvée soit dans les cellules germinales normales soit dans les tumeurs déclarées, à l'exception de la cycline $\mathrm{E}$ qui est nettement moins abondante dans le CIS que dans les séminomes [30]. Une autre caractéristique, qui est corrélée à la progression du CIS à la tumeur invasive, est la perte relative d'un inhibiteur $\mathrm{p} 18 \mathrm{INK} 4 \mathrm{C}$ kinase dépendant de la cycline qui est abondant dans le CIS mais indétectable dans les séminomes [30].

L'accumulation aberrante de la protéine p53, un autre suppresseur tumoral prototype, a été retrouvée dans les tumeurs testiculaires y compris le CIS [31]. La p53 est inactivée en raison des mutations et elle s'accumule dans un nombre élevé de tumeurs solides humaines, par exemple les tumeurs du sein et du colon. De façon surprenante, la plupart des études ne retrouvent aucune mutation du gène p53 dans les tumeurs testiculaires, ce qui indique que les mutations sont très rares (peut être en raison de l'instabilité génétique acquise durant la progression des tumeurs) et que l'accumulation de p53 est due à d'autres facteurs [32-34]. Des travaux plus récents rapportent que la forte expression de la p53 est bien corrélée à la sensibilité des cellules germinales malignes aux radiations ionisantes et à la chimiothérapie [35]. C'est pourquoi ce phénomène pourrait être caractéristique des cellules germinales en général et pourrait expliquer leur forte propension à l'apoptose en réponse à l'altération de leur ADN [36].

\section{PATHOGENÈSE DU CIS ET DES TUMEURS A CELLULE GERMINALE.}

L'origine et la pathogenèse des tumeurs à cellule germinale sont mal connues, bien que dans les années récentes ces affections aient fait l'objet d'un nombre croissant d'études épidémiologiques et moléculaires. Ces recherches ont accru notre compréhension de nombreux aspects de la carcinogenèse germinale et ont débouché sur les hypothèses actuelles concernant l'origine et la progression du CIS et des tumeurs à cellule germinale.

Il existe un consensus sur le fait que la cellule du CIS est une cellule germinale et qu'elle survient précocement dans la vie [3] ; mais le moment auquel se produit cette transformation, et le stade du développement de la cellule germinale jeune auquel elle apparaît restent encore controversés. L'incidence élevée de cancer du testicule chez les sujets avec une cryptorchidie et d'autres erreurs congénitales du 
développement gonadique, comme les ambiguïtés sexuelles (par exemple la dysgénésie gonadique), impliquent fortement la participation de facteurs intra-utérins dans la transformation maligne des cellules germinales. Une preuve indirecte complémentaire est fournie par l'analyse comparée du phénotype des cellules du CIS et des cellules germinales primordiales. Comme cité précédemment, on a identifié plusieurs marqueurs de la surface cellulaire qui différencient les cellules du CIS des cellules germinales non malignes dans le testicule post pubère. Deux protéines, de fonction et structure connues, PLAP et Kit, sont connues depuis longtemps pour être fortement exprimées par les cellules germinales primordiales des mammifères. De façon intéressante, ces deux protéines, et d'autres marqueurs du CIS comme M2A et TRA-1-60, sont exprimés par la majorité des cellules germinales foetales humaines dans le testicule pendant une très courte période, entre la 8ème et la 12ème semaine du développement [13]. Cette observation a amené l'hypothèse que durant cette période, qui peut être une étape cruciale dans la différenciation de la cellule germinale, pourrait survenir l'initiation maligne de la cellule germinale en cellule pré-CIS. Un argument supplémentaire à cette hypothèse est fourni par l'observation que dans les testicules foetaux d'individus avec ambiguités sexuelles, qui présentent un risque élevé de développement précoce de tumeurs testiculaires, l'expression de c-kit dans les cellules germinales est prolongée de façon importante [14]. La question se pose de savoir si cette étape apparemment cruciale de la différenciation de la cellule germinale est soumise à un contrôle intrinsèque absolu et ne peut être altérée que par une modification de la structure d'un gène (par exemple une mutation) ou bien si cette étape peut être influencée par des facteurs exogènes. La dysgénésie gonadique causée par un déséquilibre génétique des chromosomes sexuels est un exemple du premier scénario. D'autre part, des données épidémiologiques suggèrent qu'une influence néfaste de facteurs externes, peut-être environnementaux, soit aussi possible. Par exemple, les hommes danois nés pendant la seconde guerre mondiale ont un risque moindre de développer un cancer du testicule que les hommes nés avant ou après la guerre [37]. En outre, plusieurs études européennes ont montré que l'augmentation récente de l'incidence du cancer du testicule était principalement due à un effet cohorte, c'est à dire que l'incidence est liée à l'année de naissance plutôt qu'à l'année du diagnostic [38]. Ces observations suggèrent fortement que l'impact de facteurs externes, encore inconnus à ce jour, ait principalement pour cible les gonades immatures en développement, plus probablement au cours de la vie in utero ou dans la prime enfance [39].

Quels sont les candidats à de tels facteurs externes ? A partir de certaines observations cliniques et épidémiologiques, Handerson et son groupe ont suggéré un lien possible entre l'exposition excessive aux œstrogènes bio-disponibles in utero (associée par exemple avec la première grossesse, l'obésité maternelle, une fréquence excessive des nausées) et des anomalies reproductives chez l'homme, en particulier le cancer à cellule germinale $[40,41]$. De sévères altérations du développement gonadique, incluant les tératomes testiculaires, ont été reproduites chez des animaux de laboratoire exposés à des oestrogènes synthétiques [42]. Diverses altérations du développement gonadique mâle - dont certaines étonnamment semblables aux modifications induites soit par les oestrogènes soit par les anti-androgènes chez les animaux de laboratoire - ont été ensuite observées dans la faune sauvage, plus particulièrement dans des régions d'accumulation de polluants environnementaux [43]. Un nombre étonnamment grand de produits chimiques environnementaux très largement répandus apparaissent avoir une activité soit oestrogénique, soit anti-androgénique, et furent pour cette raison appelés "disrupteurs environnementaux" [44,45]. On avança ensuite une hypothèse selon laquelle les disrupteurs endocriniens pourraient avoir des effets néfastes sur la santé humaine en général, et plus particulièrement sur la reproduction masculine $[46,47]$. On doit insister sur le fait qu'il ne s'agit que d'une hypothèse, et qu'elle ne concerne que les populations caucasiennes. Lincidence du cancer du testicule chez les Asiatiques et chez les Africains est très faible, même chez ceux vivant dans des régions indus- 
trialisées et fortement polluées [48]. D'un autre côté, les gonades des hommes Africains pourraient être mieux protégées in utero par des taux plus élevés de testostérone chez leurs mères [49]. L'importance de la susceptibilité génétique est démontrée par de nettes différences ethniques en Europe, avec des taux d'incidence faibles en Finlande, Lituanie et Estonie par rapport à ceux des régions géographiquement proches du Danemark et de la Norvège [1]. Toutefois, l'utilisation de produits chimiques environnementaux est aussi très variable dans ces différents pays. Vu notre hypothèse qu'un retard dans le développement de la différenciation peut rendre les cellules germinales immatures plus propices à la transformation néoplasique, les effets potentiels néfastes sur le testicule en développement des disrupteurs hormonaux pourraient expliquer l'importante augmentation observée de l'incidence des cancers à cellule germinale et la décroissance concomitante des concentrations spermatiques dans les pays ayant un large recours à ces produits [50]. Toutefois, cette hypothèse nécessite d'être corroborée par de larges études épidémiologiques s'appuyant sur des expositions prouvées.

Le fait que, de façon surprenante, peu de cas familiaux de cancers testiculaires à cellule germinale aient été rapportés jusqu'alors, est cohérent avec l'hypothèse environnementale. Néanmoins, les fils et les frères d'hommes ayant développé un cancer du testicule ont respectivement 4 et 8 fois plus de risque de développer des tumeurs [51]. Jusqu'à ce jour, un seul locus pouvant correspondre à un gène de susceptibilité au cancer à cellule germinale a été rapporté sur Xq27 [52].

\section{HISTOIRE NATURELLE ET PREVA- LENCE DU CIS.}

Les études menées chez les hommes inféconds et chez ceux ayant un cancer testiculaire unilatéral avec un CIS controlatéral $[53,54]$ montrent que $50 \%$ de ces hommes, s'ils ne sont pas traités, développent une croissance invasive dans les 5 années suivantes. Le clinicien doit toutefois avoir conscience qu'à la différence d'autres types de cancer, cela ne signifie pas que les $50 \%$ restants soient spontanément résolutifs. Au contraire, si les hommes ayant un CIS sont suivis sur une plus longue période, un pourcentage croissant d'entre eux développeront une tumeur déclarée. Puisque la disparition spontanée du CIS n'a jamais été observée chez des hommes ayant eu des biopsies répétées, nous pensons que de fait tous les cas non traités de CIS se développeront en tumeurs invasives.

La prévalence du CIS dans les populations générales est basée principalement sur des évaluations statistiques grossières. La seule étude qui existe a évalué le matériel d'autopsie de gonades de 399 hommes décédés soudainement et inopinément à l'âge de 18 à 50 ans [55]. Les données recueillies à la fois dans cette étude et lors d'une réévaluation secondaire font apparaître 4 cas de CIS ou d'antécédents de tumeurs, ce qui donne une prévalence globale dans la population danoise de 1\% (4/399) (limites de l'intervalle de confiance à 95\% : $0,25-2,4 \%$ ). Connaissant la liaison entre incidence du cancer du testicule et âge, on peut présumer que cette prévalence ira en décroissant progressivement avec l'âge et sera proche de $0 \%$ entre 50 et 60 ans.

A l'opposé des rares informations sur la prévalence générale, de nombreuses études ont évalué l'incidence du CIS dans des conditions qui sont connues pour constituer un facteur de risque du cancer du testicule. Une revue de ces études a récemment été publiée par Dieckmann et Skakkebaek [56]. Un antécédent de cryptorchidie constitue probablement un groupe à risque des mieux connus de développement d'un cancer du testicule $[57,58]$. La compilation des données de plusieurs études abordant cette question indique que le CIS peut être trouvé dans les testicules d'environ $3 \%$ des hommes ayant un antécédent de cryptorchidie. Ce taux correspond à une multiplication par 3 à 5 du risque de malignité dans un testicule non descendu [58]. De plus, le risque de trouver un CIS dans une biopsie de testicule mal descendu est fortement dépendant de l'âge. Le CIS est très rarement retrouvé sur les biopsies de testicules pré-pubéres. Ceci est très probablement dû au fait que le nombre de cellules néoplasiques à cet âge est faible et que les cellules du CIS prolifèrent simultanément à 
l'augmentation pubertaire des taux de stéroïdes sexuels et de gonadotrophines. D'autre part, chez les individus au-delà de 45-50 ans, le risque de trouver un CIS est plutôt faible, puisque tous ont déjà progressé au stade de tumeur.

Un autre groupe à haut risque est constitué par les hommes ayant un cancer unilatéral du testicule. L'expérience danoise révèle une prévalence du CIS de 5 à $6 \%$ dans le testicule controlatéral $[59,60]$, ce qui est une donnée semblable aux 4,9-6,1\% récemment rapportés dans deux grandes études allemandes basées sur environ deux mille biopsies [61,62].

L'infécondité a été reliée à un risque élevé de cancer du testicule. Cependant, les hommes inféconds constituent un groupe hétérogène de maladies, comprenant des cas d'azoospermie obstructive, d'insuffisance pituitaire et de syndrome de cellules de Sertoli isolées qui ne présentent aucun risque de cancer du testicule, et des cas d'atteinte testiculaire primaire altérant la spermatogenèse qui peuvent eux représenter un vrai groupe à haut risque. Des travaux de Suisse, du Royaume Uni, d'Allemagne et du Danemark rapportent une fréquence du CIS de 0,4 à $1,1 \%$ dans les biopsies d'hommes inféconds $[63,64]$.

Un groupe rare mais important de patients à risque élevé de CIS est représenté par les individus ayant une ambiguité sexuelle avec un chromosome $\mathrm{Y}$ ou une partie de celui-ci dans leur caryotype. Une prévalence très élevée du CIS, dépassant $20-25 \%$, a été retrouvée chez les patients ayant un syndrome d'insensibilité aux androgènes, et chez les patients présentant une mosaïque $45, \mathrm{XY} / 46, \mathrm{XY}$ associée à une dysgénésie gonadique $[7,8,19]$. Enfin, la présence d'un CIS sans tumeur testiculaire déclarée ne devrait pas être négligée chez les patients ayant une tumeur à cellule germinale présumée extra-gonadique. Environ $40 \%$ de ces hommes ont un CIS dans l'un ou les deux testicules, un fait qui doit être présent à l'esprit lors de la mise en place du traitement, puisque les cellules du CIS peuvent ne pas être éradiquées par les substances cytotoxiques. De façon intéressante, le CIS n'a été retrouvé que dans les cas où on avait trouvé une tumeur rétro péritonéale soit isolée, soit associée à un élément médiastinal, mais aucun cas de CIS n'a été retrouvé chez les hommes n'ayant qu'un cancer médiastinal à cellule germinale [65].

\section{DIAGNOSTIC CLINIQUE DU CIS.}

Le CIS du testicule n'est accompagné d'aucun symptôme spécifique, bien que dans certains cas le patient puisse rapporter une certaine sensibilité de la gonade. Le testicule contenant un CIS est souvent légèrement atrophique, mais le diagnostic ne peut habituellement pas être établi sur l'examen clinique. A l'heure actuelle, la biopsie testiculaire est la seule méthode fiable de diagnostic du CIS. Une biopsie devrait être évitée si une tumeur testiculaire déclarée est palpable et présente. Une biopsie chirurgicale standard, de $3 \mathrm{~mm}$ de diamètre, pratiquée depuis de nombreuses décades dans l'évaluation de l'infécondité, est une procédure facile et sûre [66]. Une fixation appropriée des échantillons obtenus par biopsie testiculaire est d'une importance critique si on ne veut pas négliger un CIS. Le formaldéhyde tamponné, fixateur standard pour la plupart des tissus, est plutôt nuisible pour l'histologie testiculaire [67]. Les liquides de Bouin, de Stieve ou de Cleland préservent d'une façon excellente la morphologie du tissu testiculaire et permettent le diagnostic sur un échantillon coloré en routine à l'hématoxyline et à l'éosine. Le diagnostic de CIS peut en outre reposer sur la détection immunohistochimique des marqueurs du CIS tels que la PLAP.

La sensibilité de la biopsie testiculaire dans le diagnostic du CIS a été discutée, mais les données expérimentales et cliniques indiquent que cette procédure a une bonne sensibilité. Malgré le fait que des milliers de biopsies aient été réalisées, seuls 19 cas de biopsie négative ont été suivis par le développement d'un cancer $[68,56]$. Ces rares cas sont le plus souvent dûs à une distribution plus focale du CIS plutôt qu'à l'absence de cellules du CIS au moment de la biopsie. C'est pourquoi, bien que la possibilité de développement d'un cancer ne puisse être totalement exclue dans un testicule dont la biopsie est CIS négative, le patient peut être rassuré sur le fait que son risque de cancer du testicule est plus faible que celui de la population générale.

Bien que le CIS ait été diagnostiqué dans les 
testicules d'individus pré-pubères, la sensibilité de la biopsie réalisée chez les enfants est inconnue. C'est pourquoi une biopsie négative réalisée avant la puberté n'exclut pas le risque d'un futur cancer.

D'autres techniques de biopsie, comme la biopsie à l'aiguille ou l'aspiration, ont été proposées comme moyens de diagnostic du CIS $[69,70]$. La sensibilité de ces procédures dans le diagnostic de malignité précoce reste toutefois à prouver.

Des tentatives ont été faites pour développer une technique de détection des cellules du CIS dans le liquide séminal, ce qui aurait constitué un moyen non invasif de dépistage de larges populations. On pense que les cellules du CIS, comme les autres composants de l'épithélium séminifère, desquament dans le sperme. En utilisant l'immunohistochimie, l'analyse de l'ADN par cytométrie de flux et par hybridation in situ, on a mis en évidence des cellules présentant les caractéristiques de cellules germinales malignes dans le sperme d'hommes porteurs d'un CIS [71-73]. Plus récemment, la mise en évidence d'anomalies du chromosome 12 par hybridation in situ fluorescente a été utilisée avec succès pour dépister les cellules $\mathrm{du}$ CIS dans le sperme [74]. L'hybridation in situ est cependant une méthode encore trop fastidieuse et coûteuse pour être utilisée comme méthode de dépistage dans de grandes populations. Une technique idéale serait une méthode rapide et sensible assistée par PCR. Un espoir de développement d'une telle méthode de dépistage a été récemment soulevé par la découverte d'un transcrit alternatif de $1,5 \mathrm{~kb}$ du récepteur $\alpha$ du facteur de croissance dérivé des plaquettes (PDGF $\alpha$-R) dans les tumeurs malignes à cellule germinale [75]. L'intérêt de ce transcrit a été montré dans la recherche du CIS sur les biopsies transcutanées [76], bien que l'utilisation potentielle de cette technique dans l'analyse du sperme nécessite d'autres études.

L'échographie est une autre méthode non invasive qui a été utilisée pour le diagnostic du CIS. Un aspect échographique très irrégulier a été trouvé dans les testicules contenant un CIS, mais cette image échographique peut aussi être présente dans les gonades atro- phiques sans CIS. Le substrat physiopathologique à ces modifications échographiques est inconnu. Une explication partielle pourrait en être la fréquente présence de microlithes dans les testicules contenant un CIS [77]. En pratique quotidienne, l'échographie testiculaire ne peut pas être utilisée comme unique moyen de diagnostic du CIS, mais elle est habituellement d'une grande aide pour sélectionner les patients susceptibles d'avoir une biopsie testiculaire puisqu'une image échographique très irrégulière indique un risque augmenté de CIS [78,79].

En raison du risque de CIS dans le testicule controlatéral d'hommes porteurs d'une tumeur testiculaire, et de la possibilité de prévenir la survenue d'une seconde tumeur, de tels patients devraient avoir une biopsie controlatérale à la tumeur, à faire préférablement au moment de l'orchidectomie primaire. La biopsie testiculaire peut aussi être envisagée chez les hommes ayant un antécédent de cryptorchidie, principalement ceux explorés pour une infécondité. Chez les patients adressés pour orchidopexie après la puberté, une biopsie pour CIS au moment de la chirurgie devrait être considérée comme une alternative à l'orchidectomie préconisée par certains spécialistes. Tous les hommes avec une tumeur à cellule germinale extra-gonadique et ceux ayant une ambiguïté sexuelle devraient être dépistés pour le CIS en raison du risque très élevé de malignité gonadique. Les hommes inféconds sans antécédents de cryptorchidie devraient avoir une biopsie testiculaire s'ils ont des testicules atrophiques avec une image échographique irrégulière, en particulier s'il est souhaité une évaluation de la spermatogenèse en vue d'une fécondation assistée.

\section{PRISE EN CHARGE DU CIS.}

La prise en charge du CIS est différente selon l'âge des patients (enfant versus adulte) et selon la latéralité (uni versus bi) de l'atteinte. Si le CIS est unilatéral, ce qui est typiquement le cas chez les hommes inféconds et chez ceux avec antécédents de cryptorchidie, nous recommandons l'orchidectomie comme seul traitement [80]. Dans la plupart des cas, le testicule contenant le CIS contribue pour peu, voire 
rien, à la production totale de spermatozoïdes. Le testicule restant est habituellement suffisant pour maintenir une production normale d'androgènes, bien que les taux de testostérone et de LH sériques doivent être contrôlés 3 à 6 mois après l'ablation du testicule. Nous recommandons de réaliser une détermination du stade puisque chez un petit nombre d'hommes le CIS testiculaire peut s'accompagner d'une tumeur à cellule germinale extra-gonadique [65]

Les néoplasies bilatérales sont plus souvent rencontrées que le CIS dans le testicule controlatéral d'hommes ayant un cancer testiculaire unilatéral. Si le patient n'est pas traité par médication cytotoxique pour une maladie métastatique, la politique de surveillance conduira presque inévitablement au développement d'un second cancer, avec castration complète et perte de la production endogène d'androgènes [54]. C'est pourquoi, en 1985, l'irradiation localisée a été introduite comme traitement du CIS dans ce groupe d'hommes [81]. La dose initialement utilisée de $20 \mathrm{~Gy}$, répartie en 10 fractions quotidiennes égales, a permis d'éradiquer efficacement le CIS ; cependant, en raison de l'atteinte de la fonction leydigienne, cette dose a par la suite été ramenée à 14-16 Gy $[80,82]$. La radiothérapie peut être le traitement de choix dans les rares cas de CIS bilatéraux.

L'effet de l'irradiation sur les cellules germinales est démontré par l'existence d'un syndrome de cellules de Sertoli isolées sur la biopsie testiculaire de suivi, et par une concentration croissante de FSH sérique et des taux non mesurables d'inhibine B [83]. Les hommes traités par radiothérapie ont l'avantage d'avoir au moins une production de testostérone partiellement préservée. Cependant, la fertilité de ces hommes est à priori dans la majorité des cas très réduite [84-86]. Il est évident que la question de la fertilité devrait être discutée avec le patient avant une irradiation ou un traitement cytotoxique, et que la cryopréservation du sperme devrait être envisagée dans tous les cas.

Les hommes avec un CIS dans la gonade controlatérale, qui sont traités par chimiothé- rapie pour une maladie métastatique, ne devraient pas avoir une radiothérapie en première intention, mais au moins deux biopsies de surveillance devraient être réalisées chaque 1 ou 2 ans. La radiothérapie est recommandée si le CIS réapparaît à la biopsie. La chimiothérapie à base de Cisplatine peut éradiquer le CIS ; cependant, une persistance du CIS ou le développement d'un second cancer a été rapporté chez certains de ces patients $[87,88]$. En raison des nombreux effets secondaires et de l'imprévisibilité des résultats, nous ne recommandons pas la chimiothérapie comme traitement du CIS de la gonade controlatérale si un tel traitement n'est pas indiqué pour la première tumeur.

Chez les enfants pré-pubères ayant un CIS, la thérapie doit être individualisée. Ainsi, si le CIS est découvert dans un testicule cryptorchide, il peut être judicieux de répéter la biopsie peu après la puberté et de prévoir alors le traitement. Certains individus avec ambiguïté sexuelle peuvent avoir un risque très fortement élevé de développer un cancer même avant la puberté. Dans de tels cas, la décision de réaliser ou non une orchidectomie doit reposer sur la prise en compte du risque de cancer ainsi que de la croissance et de la maturation sexuelle optimales.

\section{REMARQUES CONCLUSTVES.}

Le cancer du testicule survient principalement chez les hommes jeunes à l'apogée de leur âge reproductif. L'incidence croissante de cette néoplasie, concomitante de l'incidence croissante d'autres anomalies reproductives, suggèrent une pathogenèse commune et l'implication possible de facteurs environnementaux qui, très probablement, affectent de façon défavorable le milieu hormonal de la gonade en développement. Le cancer du testicule peut être prévenu si la néoplasie est diagnostiquée au stade de CIS pré-invasif. De futures études sur le CIS testiculaire devraient non seulement apporter d'importantes informations concernant l'étiologie et la pathogenèse du cancer du testicule, mais aussi contribuer à la compréhension générale du développement et $\mathrm{du}$ fonctionnement du système reproducteur masculin. 
Remerciements : Les travaux du groupe de Copenhague cités dans cette revue ont été soutenus par des subventions de La Société de Cancérologie Danoise.

\section{REFERENCES}

1. ADAMI H, BERGSTRÖM R, MÖHNER M, ZATONSKI W, STORM H, EKBOM A, TRETLI S, TEPPO L, ZIEGLER H, RAHU M, GUREVICIUS R, STENGREVICS A. Testicular cancer in nine northern European countries. Int J Cancer 1994; 59: 33-38.

2. SKAKKEBAEK NE. Possible carcinoma in situ of the testis. Lancet 1972; 2: 516-517.

3. SKAKKEBAEK NE, BERTHELSEN JG, GIWERCMAN A, MÜLLER J. Carcinoma in situ of the testis: possible origin from gonocytes and precursor of all types of germ cell tumours except spermatocytoma. Int J Androl 1987; 10: 19-28.

4. SKAKKEBAEK NE. Atypical germ cells in the adjacent "normal" tissue of testicular tumours. APMIS 1975; 83: 127-130.

5. JACOBSEN GK, HENRIKSEN OB, VON DER MAASE H. Carcinoma in situ of testicular tissue adjacent to malignant germ-cell tumors: a study of 105 cases. Cancer 1981; 47: 2660-2662.

6. MÜLLER J, SKAKKEBAEK NE, NIELSEN OH, GREM N. Cryptorchidism and testis cancer. Atypical infantile germ cells followed by carcinoma in situ and invasive carcinoma in adulthood. Cancer 1984; 54: 629-634.

7. MÜLLER J, SKAKKEBAEK NE. Testicular carcinoma in situ in children with androgen insensitivity (testicular feminisation) syndrome. Br Med J 1984; 288: 1419-1420.

8. MÜLLER J, SKAKKEBAEK NE, RITZÉN EM, PLÖEN L, PETERSEN KE. Carcinoma in situ of the testis in children with $45, \mathrm{X} / 46, \mathrm{XY}$ gonadal dysgenesis. J Pediatr 1985; 106: 431-436.

9. MANIVEL JC, JESSURUN J, WICK MR, DEHNER LP. Placental alkaline phosphatase immunoreactivity in testicular germ-cell neoplasms. Am J Surg Pathol 1987; 11: 21-29.

10. HOFMANN M-C, JELTSCH W, BRECHER J, WALT H. Alkaline phosophatase isozymes in human testicular germ cell tumors, their precancerous stage, and three related cell lines. Cancer Res 1989; 49: $4696-4700$.

11. STROHMEYER T, PETER S, HARTMANN M, MUNEMITSU S, ACKERMANN L, ULLRICH A, SLAMON DJ. Expression of the hst-1 and c-kit protooncogenes in human testicular germ cell tumors. Cancer Res 1991; 51: 1811-1816.

12. RAJPERT-DE MEYTS E, SKAKKEBAEK NE. Expression of the c-kit protein product in carcinoma in situ and invasive testicular germ cell tumours. Int J Androl 1994;17:85-92.

13. JØRGENSEN N, RAJPERT-DE MEYTS E, GRÆM N, MÜLLER J, GIWERCMAN A, SKAKKEBAEK NE. Expression of immunohistochemical markers for testicular carcinoma in situ by normal human foetal germ cells. Lab Invest 1995; 72: 223-231.

14. RAJPERT-DE MEYTS E, JøRGENSEN N, MÜLLER J, SKAKKEBAEK NE. Prolonged expression of the c-kit receptor in germ cells of intersex fetal testes. J Pathol 1996; 178: 166-169.

15. GIWERCMAN A, CANTELL L, MARKS A. Placentallike alkaline phosphatase as a marker of carcinoma in situ of the testis. Comparison with monoclonal antibodies M2A and 43-9F. APMIS 1991; 99: $586-594$.

16. GIWERCMAN A, ANDREWS PW, JøRGENSEN N, MÜLLER J, GRAEM N, SKAKKEBAEK NE. Immunohistochemical expression of embryonal marker TRA-1-60 in carcinoma in situ and germ cell tumors of the testis. Cancer 1993; 72: 1308-1314.

17. KANG J-L, RAJPERT-DE MEYTS E, WIELS J, SKAKKEBAEK NE. Expression of the glycolipid globotriaosylceramide $(\mathrm{Gb} 3)$ in testicular carcinoma in situ. Virchows Arch 1995; 426: 369-374.

18. RAJPERT-DE MEYTS E, KVIST M, SKAKKEBAEK $\mathrm{NE}$. Heterogeneity of expression of immunohistochemical tumour markers in testicular carcinoma in situ : pathogenetic relevance. Virchovs Arch 1996; 428: 133-139.

19. MÜLLER J. Abnormal infantile germ cells and development of carcinoma in situ in maldeveloped testes: a stereological and densitometric study. Int J Androl 1987; 10: 543-567.

20. ATKIN NB, BAKER MC. i(12p): specific chromosomal marker in seminoma and malignant teratoma of the testis? Cancer Genet Cytogenet 1983;10:199-204.

21. VAN ECHTEN J, OOSTERHUIS JW, LOOIJENGA LHJ, WIERSMA J, TE MEERMAN G, SCHRAFFORDT-KOOPS H, SLEIJFER DT, DE JONG B. No recurrent structural abnormalities in germ cell tumors of the adult testis apart from i(12p). Genes Chromosomes Cancer 1995;14:133-144.

22. VAN KESSEL AG, SUIJKERBRUIJK RF, SINKE RJ, LOOIJENGA L, OOSTERHUIS JW, DE JONG B. Molecular cytogenetics of human germ cell tumours: i(12p) and related chromosomal anomalies. Eur Urol 1993;23:23-29.

23. LOOIJENGA LHJ, GILLIS AJM, VAN PUTTEN WLJ, OOSTERHUIS JW. In situ numeric analysis of centromeric regions of chromosome 1, 12, 15 of seminomas, nonseminomatous germ cell tumors and carcinoma in situ of human testis. Lab Invest 1993;68:211-219.

24. VAN ECHTEN J, VAN GURP RJHLM, STOEPKER M, LOOIJENGA LHJ, DE JONG B, OOSTERHUIS 
JW. Cytogenetic evidence that carcinoma in situ is the precursor lesion for invasive testicular germ cell tumours. Cancer Genet Cytogenet 1995; 85: 133-137.

25. STROHMEYER TG, SLAMON DJ. Proto-oncogenes and tumor suppressor genes in human urological malignancies. J Urol 1994; 151: 1479-1497.

26. MURTY VVNS, HOULDSWORTH J, BALDWIN S, REUTER V, HUNZIKER W, BESMER P, BOSL G, CHAGANTI RSK. Allelic deletions in the long arm of chromosome 12 identify sites of candidate tumor suppressor genes in male germ cell tumors. Proc Natl Acad Sci USA 1992;89:11006-11010.

27. HOULDSWORTH J, REUTER V, BOSL GJ, CHAGANTI RSK. Aberrant expression of cyclin D2 is an early event in human male germ cell tumorigenesis. Cell Growth \& Differentiation 1997;8: 293-299.

28. BARTKOVA J, RAJPERT-DE MEYTS E, SKAKKEBAEK NE, BARTEK J. D-type cyclins in adult human testis and testicular cancer: Relation to cell type, proliferation, differentiation, and malignancy. J Pathol 1999; 187: 573-81.

29. STROHMEYER T, REISSMANN P, CORDONCARDO C, HARTMANN M, ACKERMANN R, SLAMON D. Correlation between retinoblastoma gene expression and differentiation in human testicular tumors. Proc Natl Acad Sci USA 1991; 88: $6662-6666$.

30. BARTKOVA J, THULLBERG M, RAJPERT-DE MEYTS E, SKAKKEBAEK NE, BARTEK J. Cell cycle regulators in testicular cancer: loss of p18INK4C marks progression from carcinoma in situ to invasive germ cell tumours. Int J Cancer 2000; 85: 370-5.

31. BARTKOVA J, BAR'TEK J, LUKAS J, VOJTESEK B, STASKOVA Z, REJTHAR A, KOVARIK J, MIDGLEY CA, LANE DP. p53 protein alterations in human testicular cancer including pre-invasive intratubular germ-cell neoplasia. Int J Cancer 1991; 48: 196-202.

32. KUCZYK MA, SERTH J, BOKEMEYER C, JONASSEN J, MACHTENS S, WERBER M, JONAS U. Alterations of the p53 tumor suppressor gene in carcinoma in situ of the testis. Cancer 1996: 78; 19581966.

33. HEIMDAL K, LOTHE RA, LXSTAD S, HOLM R, FOSSA SD, BØRRESEN A-L. No germline TP 53 mutations detected in familial and bilateral testicular cancer. Genes Chromosomes Cancer 1993; 6: 9297.

34. PENG HQ, HOGG D, MALKIN D, BAILEY D, GALLIE BL, BULBUI M, JEWETT M, BUCHANAN J, GOSS PE. Mutations of the p53 gene do not occur in testis cancer. Cancer Res 1993; 53: 3574-3578.

35. CHRESTA CM, MASTERS JRW, HICKMAN JA. Hypersensitivity of human testicular tumors to etoposide-induced apoptosis is associated with functional p53 and high bax:bcl-2 ratio. Cancer Res 1996; 56: 1834-1841.
36. LUTZKER SG. Regulation of tumour suppressor activity in teratocarcinoma cells. APMIS 1998; 106: 8589.

37. MØLLER H. Clues to the aetiology of testicular germ cell tumours from descriptive epidemiology. Eur Urol 1993; 23: 8-15.

38. BERGSTRÖM R, ADAMI HO, MÖHNER M, ZATONSKI W, STORM H, TRETLI S, TEPPO L, AKRE O, HAKULINEN T. Increase in testicular cancer incidence in six European countries: a birth cohort phenomenon. J Natl Cancer Inst 1996; 88: 727-733.

39. RAJPERT-DE MEYTS E, JØRGENSEN N, BRØNDUM-NIELSEN K, MÜLLER J, SKAKKEBAEK NE. Developmental arrest of germ cells in the pathogenesis of germ cell neoplasia. APMIS 1998; 106: 198-204.

40. HENDERSON BE, ROSS RK, PIKE MC, CASAGRANDE JT. Endogenous hormones as a major factor in human cancer. Cancer Res 1982; 42: 3232-3239.

41. DEPUE RH, PIKE MC, HENDERSON BE. Estrogen exposure during gestation and risk of testicular cancer. J Natl Cancer Inst 1983; 71: 1151-1155.

42. MCLACHLAN JA, NEWBOLD RR, BULLOCK B. Reproductive tract lesions in male mice exposed prenatally to diethylstilbestrol. Science 1975; 190: 991992.

43. GUILETTE LJ, GROSS TS, MASSON GR, MATTER JM, PERCIVAL HF, WOODWARD AR. Developmental abnormalities of the gonad and abnormal sex hormone concentrations in juvenile alligators from contaminated and control lakes in Florida. Environ Health Perspect 1994; 102: $680-688$.

44. SOTO AM, CHUNG KL, SONNENSCHEIN C. The pesticides endosulfan, toxaphene, and dieldrin have estrogenic effects on human estrogen sensitive cells. Environ Health Perspect 1994; 102: 380-386.

45. KELCE WR, STONE CR, LAWS SC, GRAY LEJ, KEMPPAINEN JA, WILSON EM. Persistent DDT metabolite, p,p'-DDE is a potent androgen receptor antagonist. Nature 1995; 375: 581-585.

46. SHARPE RM, SKAKKEBAEK NE. Are oestrogens involved in falling sperm counts and disorders of the male reproductive tract? Lancet 1993; 341: 1392-1395.

47. TOPPARI J, LARSEN JC, CHRISTIANSEN P, GIWERCMAN A, GRANDJEAN P GUILLETTE LJ, JÉGOU B, JENSEN TK, JOUANNET P, KEIDING $\mathrm{N}$, LEFFERS $\mathrm{H}$, MCLACHLAN JA, MEYER $\mathrm{O}$, MÜLLER J, RAJPERT-DE MEYTS E, SCHEIKE T, SHARPE R, SUMPTER J, SKAKKEBAEK NE. Male reproductive health and environmental xenoestrogens. Environ Health Perspect 1996; 104(Suppl 4): 741-803.

48. VAN DEN EEDEN SK, WEISS NS. Is testicular can- 
cer incidence in blacks increasing? Am J Public Health 1989;79:1553-1554.

49. HENDERSON BE, BERNSTEIN L, ROSS RK, DEPUE RH, JUDD HL. The early in utero oestrogen and testosterone environment of blacks and whites: potential effects on male offspring. $\mathrm{Br} \mathrm{J}$ Cancer 1988; 57: 216-218.

50. SKAKKEBAEK NE, RAJPERT-DE MEYTS E, JØRGENSEN N, CARLSEN E, PETERSEN PM, GIWERCMAN A, ANDERSEN AG, JENSEN TK, ANDERSSON A-M, MÜLLER J. Germ cell cancer and disorders of spermatogenesis: An environmental connection? APMIS 1998; 106: 3-12.

51. FORMAN D, OLIVER RTD, BRETT AR, MARSH SGE, MOSES JH, BODMER JG, CHILVERS CED, PIKE MC. Familial testicular cancer: a report of the UK family register estimation of risk and HLA class I sibpair analysis. Br J Cancer 1992; 65: 255-262.

52. RAPLEY EA, CROCKFORD GP, TEARE D, BIGGS P, SEAL S, BARFOOT R, EDWARDS, S, HAMOUDI R, HEIMDAL K, FOSSÅ SD, TUCKER K, DONALD J, COLLINS F, FRIEDLANDER M, HOGG D, GOSS P, HEIDENREICH A, ORMISTON W, DALY PA, FORMAN D, OLIVER RTD, LEAHY M, HUDDART R, COOPER CS, BODMER JG, EASTON DF, STRATTON MR, BISHOP DT. Localization to Xq27 od a susceptibility gene for testicular germ-cell tumours. Nature Genetics 2000; 24: 197-200.

53. SKAKKEBAEK NE, BERTHELSEN JG, MÜLLER J. Carcinoma in situ of the undescended testis. Urol Clin North Am 1982; 9: 377-385.

54. VON DER MAASE H, RØRTH M, WALBOM-JØRGENSEN S, SØRENSEN BL, CHRISTOPHERSEN IS, HALD T, JACOBSEN GK, BERTHELSEN JG, SKAKKEBAEK NE. Carcinoma in situ of contralateral testis in patients with testicular germ cell cancer: study of 27 cases in 500 patients. Br Med J 1986; 293: 1398-1401.

55. GIWERCMAN A, MÜLLER J, SKAKKEBAEK NE. Prevalence of carcinoma in situ and other histopathological abnormalities in testes from 399 men who died suddenly and unexpectedly. J Urol 1991; 145: 77-80.

56. DIECKMANN K-P, SKAKKEBAEK NE. Carcinoma in situ of the testis: review of biological and clinical features. Int J Cancer 1999; 83: 815-822.

57. GILBERT JB, HAMILTON JB. Studies in malignant testis tumors. III. Incidence and nature of tumors in ectopic testes. Surg Gynecol Obstet 1940; 71: 731-743.

58. GIWERCMAN A, GRINDSTED J, HANSEN B, JENSEN OM, SKAKKEBAEK NE. Testicular cancer risk in boys with maldescended testis: a cohort study. J Urol 1987; 138: 1214-1216.

59. BERTHELSEN JG, SKAKKEBAEK NE, VON DER MAASE H, SøRENSEN BL, MOGENSEN P. Screening for carcinoma in situ of the contralateral testis in patients with germinal testicular cancer. $\mathrm{Br}$ Med J 1982; 285: 1683-1686.

60. VON DER MAASE H, RØRTH M, WALBOM-JØRGENSEN $S$ et al. Carcinoma in situ of the contralateral testis in patients with testicular germ cell cancer: study of 27 cases in 500 patients. Br Med J 1986; 293: 1398-1401.

61. DIECKMANN K-P, LOY V. Prevalence of contralateral testicular intraepithelial neoplasia in patients with testicular germ cell neoplasms. J Clin Oncol 1996; 14: 3126-3132.

62. HOLSTEIN AF, LAUKE $\mathrm{H}$. Histologic diagnostics of early testicular germ cell tumour. Int J Urol 1996; 3: 165-172.

63. PRYOR JP, CAMERON KM, CHILTON CP, et al. Carcinoma in situ in testicular biopsies from men presenting with infertility. $\mathrm{Br} \mathrm{J}$ Urol 1983; 55: $780-784$.

64. GIWERCMAN A, THOMSEN JK, HERTZ J, BERTHELSEN JG, JENSEN V, MEINECKE B, THORMANN L, STORM HH, SKAKKEBAEK NE. Prevalence of carcinoma in situ of the testis in 207 oligozoospermic men from infertile couples: prospective study of testicular biopsies. Br Med J 1997; 315 : 989-991.

65. DAUGAARD G, RØRTH M, VON DER MAASE $H$, SKAKKEBAEK NE. Management of extragonadal germ-cell tumors and the significance of bilateral testicular biopsies. Ann Oncol 1992; 3: 283-289.

66. BRUUN E, FRIMODT-MøLLER C, GIWERCMAN A, LENZ S, SKAKKEBAEK NE. Testicular biopsy as an outpatient procedure in screening for carcinoma in situ: complications and the patient's acceptance. Int J Androl 1987; 10: 199-202.

67. SCHÜTTE B. The importance of fixatives for detection of carcinoma in situ (CIS) in testicular tissue. Andrologia 1988; 20: 422-425.

68. DIECKMANN K-P, KAUP F, LOY V. False-negative biopsy for testicular intraepithelial neoplasia. J Cancer Res Clin Oncol 1993; 119: 1-4.

69. NAGLER HM, KAUFMAN DG, O'TOOLE KM, SAWCZUK IS. Carcinoma in situ of the testes: diagnosis by aspiration flow cytometry. J Urol 1990; 143: 359-361.

70. BRACKENBURY ET, HARGREAVE TB, HOWARD GCW, MCINTYRE MA. Seminal fluid analysis and fine-needle aspiration cytology in the diagnosis of carcinoma in situ of the testis. Eur Urol 1993; 23: $123-128$.

71. GIWERCMAN A, CLAUSEN OPF, SKAKKEBAEK NE. Carcinoma in situ of the testis: aneuploid cells in semen. Br Med J 1988; 296: 1762-1764.

72. GIWERCMAN A, MARKS A, SKAKKEBAEK NE. Carcinoma in situ germ cells exfoliated from seminiferous epithelium into seminal fluid. Lancet 1988; 1: 530-532. 
73. GIWERCMAN A, HOPMAN AHN, RAMAEKERS FCS, SKAKKEBAEK NE. Carcinoma in situ of the testis. Detection of malignant germ cells in seminal fluid by means of in situ hybridization. Am J Pathol 1990; 136: 497-502.

74. MENG FJ, ZHOU Y, GIWERCMAN A, SKAKKEBAEK NE, GEURTS VAN KESSEL A, SUIJKERBUIJK R. Fluorescence in situ hybridization analysis of chromosome 12 anomalies in semen cells from patients with carcinoma in situ of the testis. J Pathol 1998; 186: 235-239.

75. MOSSELMAN S, LOOIJENGA LHJ, GILLIS AJM, VAN ROOIJEN MA, KRAFT HJ, VAN ZOELEN EJJ, OOSTERHUIS JW. Aberrant platelet-derived growth factor a-receptor transcript as a diagnostic marker for early human germ cell tumors of the adult testis. Proc Natl Acad Sci USA 1996; 93: 2884-2888.

76. OOSTERHUIS JW, GILLIS AJM, VAN ROOZENDAAL CEP, VAN ZOELEN EJJ, LOOIJENGA LHJ. The platelet-derived growth factor a-receptor $1.5 \mathrm{~kb}$ transcript: target for molecular detection of testicular germ cell tumours of adolescents and adults. APMIS 1998; 106: 207-215.

77. KANG J-L, RAJPERT-DE MEYTS E, GIWERCMAN A, SKAKKEBAEK NE. The association of testicular carcinoma in situ with intratubular microcalcifications. J Urol Pathol 1994; 2: 235-242.

78. LENZ S, GIWERCMAN A, SKAKKEBAEK NE, BRUUN E, FRIMODT-MØLLER C. Ultrasound in detection of early neoplasia of the testis. Int J Androl 1987; 10: 187-190.

79. GIWERCMAN A, LENZ S, SKAKKEBAEK NE. Carcinoma in situ in atrophic testis selected for biopsy by abnormal ultrasound pattern. Br J Urol 1993; 72: $118-120$.

80. VON DER MAASE H, GIWERCMAN A, MÜLLER J, SKAKKEBAEK NE. Management of carcinoma in situ of the testis. Int J Androl 1987; 10: 209-220.

81. VON DER MAASE H, GIWERCMAN A, SKAKKEBAEK NE. Radiation treatment of carcinoma in situ of testis. Lancet 1986; 1: 624-625.

82. GIWERCMAN A, VON DER MAASE H, BERTHELSEN JG, RØRTH M, BERTELSEN A, SKAKKEBAEK NE. Localized irradiation of testes with carcinoma in situ: effects on Leydig cell function and eradication of malignant germ cells in 20 patients. J Clin Endocrinol Metab 1991; 73: 596-603.

83. PETERSEN PM, ANDERSSON AM, RøRTH M, DAUGAARD G, SKAKKEBAEK NE. Undetectable Inhibin B serum levels in men after testicular irradiation. J Clin Endocrinol Metab 1999; 84; 213-215.

84. GIWERCMAN A, VON DER MAASE H, RØRTH M, SKAKKEBAEK NE. Semen quality in testicular tumour and CIS in the contralateral testis. Lancet 1993; 341: 384-385.

85. FORDHAM MVP, MASON MD, BLACKMORE C,
HENDRY WF, HORWICH A. Management of the contralateral testis in patients with testicular germ cell cancer. Br J Urol 1990; 65: 290-293.

86. PETERSEN PM, GIWERCMAN A, HANSEN SW, BERTHELSEN JG, DAUGAARD G, RØRTH M, SKAKKEBAEK NE. Impaired testicular function in patients with carcinoma-in-situ of the testis. J Clin Oncol 1999; 17: 173-179.

87. VON DER MAASE H, MEINECKE B, SKAKKEBAEK NE. Residual carcinoma in situ of contralateral testis after chemotherapy. Lancet 1988;1:477-478.

88. FOSSA SD, AASS N. Cisplatin-based chemotherapy does not eliminate the risk of a second testicular cancer. Br J Urol 1989; 63: 531-534.

89. Parkin DM, Whelan SL, Ferlay J, Raymond L, Young $\mathrm{J}$ (eds). Cancer in five continents, volume VII. Lyon, International Agency for Research on Cancer Scientific Publications No. 143, 1997.

\section{ABSTRACT}

Carcinoma in Situ of the Testis - a Precursor of Germ Cell Cancer.
A Review of Pathogenesis and Clinical Aspects.

\section{E. RAJPERT-DE MEYTS, A. GIWERCMAN, N. E.SKAKKEBAEK}

Testicular germ cell tumors are different than other solid tumours with regard to their epidemiology and certain biological aspects. Pathogenesis of this malignancy remains unknown with the exception that all types of germ cell tumours in young adults originate from a common precursor: carcinoma in situ (CIS) cell. A recent dramatic increase in the incidence of testicular cancer suggests that environmental factors, especially those acting during development, may play a role. This review summarises the most important biological features of testicular CIS and discusses clinical management.

Key Words: testicular neoplasm, carcinoma in situ, germ cell, gonadal development, male reproduction. 